\title{
CIRANO
}

Centre interuniversitaire de recherche

en analyse des organisations

RAPPORT BOURGOGNE

\section{SYSTÈMES D'INFORMATION INTER-ORGANISATIONNELS}

Benoit A. Aubert

Fellow, CIRANO

Professeur agrégé, HEC Montréal

\section{AYMERIC DUSSART}

MSc, HEC Montréal

Analyste Conseil, Robichaud Conseil Inc.

(Mars 2002) 


\section{Les Rapports Bourgogne}

Documents de synthèse portant sur des questions d'intérêt général produits par des Fellows CIRANO, les Rapports bourgogne contribuent à alimenter la réflexion et le débat public sur des questions d'actualité. Les idées et les opinions émises dans ces rapports sont sous l'unique responsabilité des auteurs, et ne représentent pas nécessairement les positions du CIRANO ou de ses partenaires corporatifs, universitaires et gouvernementaux.

\section{The Burgundy Reports}

The Burgundy Reports are written by CIRANO Fellows on issues of general interest, and aim at encouraging discussion and debate. The observations and viewpoints expressed are the sole responsibility of the authors; they do not necessarily represent positions of CIRANO or its corporative, university or governmental partners.

\section{CIRANO}

Le CIRANO est un organisme sans but lucratif constitué en vertu de la Loi des compagnies du Québec. Le financement de son infrastructure et de ses activités de recherche provient des cotisations de ses organisations-membres, d'une subvention d'infrastructure du ministère de la Recherche, de la Science et de la Technologie, de même que des subventions et mandats obtenus par ses équipes de recherche.

CIRANO is a private non-profit organization incorporated under the Québec Companies Act. Its infrastructure and research activities are funded through fees paid by member organizations, an infrastructure grant from the Ministère de la Recherche, de la Science et de la Technologie, and grants and research mandates obtained by its research teams.

\section{Les organisations-partenaires / The Partner Organizations}

École des Hautes Études Commerciales

École Polytechnique

Université Concordia

Université de Montréal

Université du Québec à Montréal

Université Laval

Université McGill

Ministère des Finances du Québec

MRST

Alcan inc.

AXA Canada

Banque du Canada

Banque Laurentienne du Canada

Banque Nationale du Canada

Banque Royale du Canada

Bell Québec

Bombardier

Bourse de Montréal

Développement des ressources humaines Canada [DRHC]

Fédération des caisses Desjardins du Québec

Hydro-Québec

Industrie Canada

Pratt \& Whitney Canada Inc.

Raymond Chabot Grant Thornton

Ville de Montréal

(C) 2002 Benoit Aubert et Aymeric Dussart. Tous droits réservés. All rights reserved.

Reproduction partielle permise avec citation du document source, incluant la notice ${ }^{\circ}$.

Short sections may be quoted without explicit permission, provided that full credit, including $\odot$ notice, is given to the source. 


\section{TABLE DES MATIÈRES}

\begin{tabular}{ll}
\hline 1. Introduction & $\mathbf{5}$ \\
\hline 1.1 Définition & 6 \\
1.2 Pourquoi les systèmes d'information inter-organisationnels & 8 \\
1.3 La classification des SIIO & 9 \\
Illustration : le portail de l'automobile covisint & 10 \\
\hline 2. Information partagée : Les hubs électroniques et & $\mathbf{1 2}$ \\
les places de marché & \\
\hline 2.1 Les places de marché : «extranets on steroids» & 12 \\
2.2 Fonctions et caractéristiques & 12 \\
2.3 Joindre ou ne pas joindre une place de marché & 14 \\
\hline 3. Interdépendance Séquentielle: Systèmes d'information \\
inter-organisationnels et chaîne d'approvisionnement & $\mathbf{1 6}$ \\
\hline 3.1 Concepts de chaîne d'approvisionnement & 16 \\
3.2 Systèmes d'information en support à la chaîne de valeur ou à & 19 \\
la chaîne d'approvisionnement & \\
3.3 Synthèse des technologies & 28 \\
\hline 4. Interdépendance réciproque : SIIO Réseau & $\mathbf{2 9}$ \\
\hline 4.1 Introduction au travail en collaboration & 29 \\
4.2 Les systèmes de gestion de workflows & 30 \\
4.3 Édition commune de documents & 32 \\
4.4 Synthèse des technologies & 33 \\
\hline 5. Conclusion & $\mathbf{3 4}$ \\
\hline 5.1 Considérations opérationnelles & 34 \\
5.2 Considérations stratégiques & 35 \\
5.3 Risques associés & 37 \\
5.4 Remarques finales & 39 \\
\hline
\end{tabular}





\section{Introduction}

"I strongly believe that the real power of IT for any firm lies not in streamlining internal operations (efficiency enhancements) but in restructuring the relationship in the extended business networks to leverage a broader array of competencies that will deliver superior products and services"

[N. Venkatraman, 1994, $\mathrm{p} 83^{1}$ ]

D'abord introduites dans les années 60 comme outils d'automatisation des tâches comptables, les technologies de l'information se sont généralisées dans les années 70 avec les tâches opérationnelles. Les années 80 ont ensuite été marquées par le end-user computing avec l'arrivée de l'ordinateur personnel dans l'organisation. Cette période fut celle de l'information destinée à la prise de décision. Le début des années 90 a ensuite été une période caractérisée par un certain scepticisme vis à vis des technologies de l'information (TI). En effet, malgré l'évolution des technologies et l'importance des investissements réalisés, on ne mesurait toujours pas des gains en performance notables et tout particulièrement en terme de productivité.

Plusieurs travaux furent alors publiés en cette même période pour promouvoir de profonds changements organisationnels lors de l'implantation de systèmes dans les organisations. Les TI ne devaient plus être de simples outils d'automatisation mais devaient être utilisées pour mettre fin aux divisions des organisations par fonctions. L'ère du Re-engineering et des systèmes intégrés tels que SAP R/3, Peoplesoft et autres débutait. L'implantation de ces systèmes connut notamment un sommet à la fin des années 90 lorsque ces derniers ont permis de remplacer des systèmes hérités pour lesquels le passage à l'an 2000 aurait entraîné des mises à jour coûteuses.

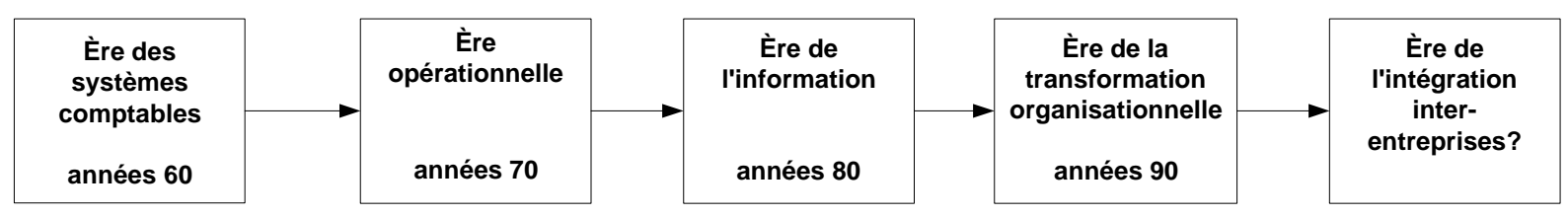

Figure 1 - Cinq ères en technologies de l’information

\footnotetext{
${ }^{1}$ Venkatraman N., " IT-Enabled Business Transformation: From Automation to Business Scope Redefinition ”, Sloan Management Review, Winter 1994, pp. 73-87.
} 
À ce jour, pour beaucoup d'organisations, cette phase d'intégration intraorganisationnelle est complétée ou est en bonne voie de l'être. L'intérêt se tourne

L'intérêt se tourne maintenant vers une intégration interorganisationnelle où de nombreux gains en terme d'efficacité sont espérés.

Un système d'information inter-organisationnel (SIIO) a pour fonction particulière de supporter des processus qui traversent les frontières d'une organisation. donc maintenant vers une intégration inter-organisationnelle où de nombreux gains en terme d'efficacité sont espérés. En effet, en 2002, une grande partie des échanges entre organisations n'est pas encore automatisée et se fait par des moyens traditionnels tels que le téléphone, le fax ou le courrier.

L'objectif de ce rapport est de présenter un ensemble de technologies, d'exemples concrets d'implantation et enfin de concepts reliés aux systèmes d'information inter-organisationnels (SIIO) afin de clarifier un peu la situation vis à vis ce sujet.

\subsection{Définition}

Un système d'information inter-organisationnel (SIIO) a pour fonction particulière de supporter des processus qui traversent les frontières d'une organisation. Il peut ainsi supporter des processus étroitement couplés entre deux organisations. Illustrons ce cas par un exemple très simple de processus de prise de commande d'un manufacturier à un sous-traitant qui doit accuser réception de la commande :

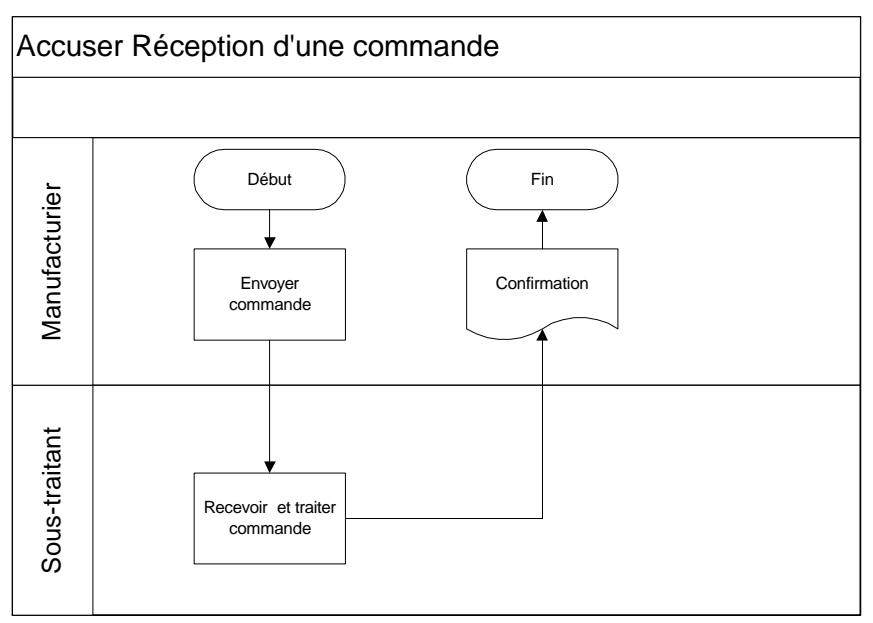

Figure 2 - Processus de prise de commande 
On remarque que ce processus pourrait être supporté de plusieurs façons différentes. On pourrait utiliser une architecture qui envoie un message du système d'une organisation à l'autre. On pourrait utiliser le système de prise de commande web du sous-traitant. Ou inversement, le sous-traitant pourrait constater par lui-même via l'extranet du manufacturier que ce dernier a un niveau des stocks sous le seuil de réapprovisionnement. Il existe différentes formes possibles d'inter-opératibilité pour des processus d'affaires automatisés, que l'on nomme également workflows. Les cinq plus connues sont les suivantes :

- Le partage de capacité : L'exécution des deux processus (de chaque partenaire) est supportée par un système unique qui n'est pas propre à une seule organisation. Un système inter-organisationnel de travail collaboratif où les tâches sont distribuées entre plusieurs organisations en est une illustration.

- L'exécution en chaîne : L'inter-opérabilité des processus est séquentielle. Le processus d'affaires inter-organisationnel est découpé en sous-processus intra-organisationnels qui sont exécutés séquentiellement par un système d'information différent dans chaque organisation composant ainsi globalement un système d'information inter-organisationnel.

- Sous-traitance : Un des partenaires commerciaux sous-contracte des sousprocessus à d'autres organisations. Il peut s'agir pour un manufacturier de sous-contracter par exemple la production ou la livraison. Pour le manufacturier, l'opération n'apparaît que comme une activité mais pour le partenaire, il s'agit d'un processus complexe. Un exemple de ce genre de situation est offert par United Parcel Service (UPS) qui offre des services d'impartition du processus de livraison.

- Transfert de cas : Le transfert de cas correspond à une situation où chaque partenaire d'affaires possède une copie de la définition du processus et chaque instance du processus peut être transférée d'une organisation à une autre pour équilibrer la charge de travail. Les places de marché électroniques qui intègrent des sous-traitants de bas niveaux illustrent un tel cas. En effet, ces sous-traitants peuvent posséder des capacités de production plus limitées mais possèdent souvent des processus très similaires. La place de marché permet alors de répartir les commandes de façon plus équilibrée.

\section{Cinq workflows possibles :}

- Partage de capacité

- Exécution en chaîne

- Sous-traitance

- Transfert de cas

- Faiblement couplée 
- Faiblement couplée : La dernière forme possible d'architecture est probablement la plus utilisée. Dans cette forme, le processus est découpé en parties qui peuvent être actives en parallèle et non uniquement séquentiellement. Le processus interne est alors purement propriétaire, seuls les protocoles de communications sont publics. Chaque partenaire peut modifier son processus dans la mesure où il ne modifie en rien le protocole de communication. Une illustration moderne est un système EDI où le processus interne d'un manufacturier tel que Bombardier est généralement ignoré du sous-traitant qui reçoit électroniquement des bons de commandes venant de ce dernier.

\subsection{Pourquoi des systèmes inter-organisationnels?}

Les systèmes d'information inter-organisationnels sont de plus en plus présents dans les organisations parce que la façon de gérer les entreprises changent. De plus en plus, certaines activités d'un processus d'affaires d'une organisation dépendent d'une activité effectuée à l'extérieur de ses frontières. Auparavant, les organisations

De plus en plus d'organisations adoptent des structures plus légères, se concentrant sur les activités proches de leurs compétences de base et confiant à des partenaires le reste de leurs activités. hiérarchiques avaient un nombre limité d'interactions avec leurs partenaires en amont et en aval. Les commandes étaient passées à intervalles réguliers (souvent annuellement) et généralement en grandes quantités. Ce modèle change radicalement dès les années 1990. On voit de plus en plus d'organisations adopter des structures plus légères, se concentrant sur les activités proches de leurs compétences de base et confiant à des partenaires le reste de leurs activités. Ces entreprises ont dès lors un nombre nettement plus important d'échanges d'information avec leurs partenaires que les entreprises traditionnelles.

Par exemple, on a vu apparaître depuis environ dix ans des organisations réseau (comme Nike, SuperBakery ou Li \& Fung) où les frontières de la firme se limitent à encadrer les activités fondamentales, que ce soit au niveau de l'administration, au niveau de la recherche et du développement, ou au niveau du contrôle de l'interface avec le client. L'entreprise conserve strictement son noyau. Les autres fonctions de l'entreprise sont imparties. Ainsi, en plus des activités classiques d'achat et de vente, les SIIO peuvent être utilisés pour faciliter l'impartition, que ce soit au niveau de la négociation ou au niveau du travail collaboratif réalisé une fois un partenaire choisi. Ces systèmes permettent à l'organisation de se coordonner avec ses 
partenaires. Grâce aux innovations technologiques, les nouvelles formes d'organisation deviennent plus attrayantes (en fait, elles deviennent possibles). La coordination serrée entre les entreprises devient quelque chose de réalisable à un coût raisonnable.

\subsection{La classification des SIIO}

Nous allons, dans ce rapport, présenter les différents types de SIIO en utilisant une classification basée sur le type d'interdépendance existant entre les différentes organisations utilisant le système ${ }^{2}$. Par “ interdépendance ", on cherche à déterminer de quelle façon le travail d'une organisation est dépendant de celui d'une autre (ou le travail de deux organisations est interdépendant). Trois formes d'interdépendances et donc de SIIO sont identifiés. Elles sont présentées dans le tableau suivant.
Grâce aux innovations technologiques, la coordination serrée entre les entreprises devient réalisable à un coût raisonnable.

\begin{tabular}{|c|c|c|c|}
\hline $\begin{array}{c}\text { Type de système } \\
\text { d'information inter- } \\
\text { organisationnel (SIIO) }\end{array}$ & $\begin{array}{c}\text { SIIO d'information } \\
\text { partagée }\end{array}$ & $\begin{array}{c}\text { SIIO de chaîne } \\
\text { d'approvisionnement } \\
\text { / de valeur }\end{array}$ & SIIO réseau \\
\hline Type d'interdépendance & Groupée & Séquentielle & Réciproque \\
\hline Configuration & & & \\
\hline Potentialité de conflit & Faible & Moyenne & Importante \\
\hline Exemples de systèmes & $\begin{array}{c}\text { Partage d'un } \\
\text { Extranet } \\
\text { Place de marché } \\
\text { électronique }\end{array}$ & $\begin{array}{c}\text { Systèmes transactionnels EDI } \\
\text { Systèmes transactionnels XML }\end{array}$ & Groupware \\
\hline
\end{tabular}

\footnotetext{
${ }^{2}$ Cette classification a été propose par Kumar, K. etVan Dissel, H.G., "Sustainable Collaboration : Managing Conflict and Cooperation in Interorganizational Systems " MIS Quarterly, Sep 1996, 20(3), 279-300.
} 
La première forme d'interdépendance consiste en un partage collectif d'information et sa forme la plus connue est la place de marché électronique ("e-marketplaces"). La deuxième forme d'interdépendance est séquentielle et correspond aux SIIO en support à la chaîne d'approvisionnement tels que l'EDI ou les systèmes de prise de commande. Enfin, la troisième forme correspond à une interdépendance de type réseau et correspond aux SIIO pour le travail en collaboration tels que les groupwares. Ces formes utilisent une variété d'outils.

Chacune de ces formes de SIIO sera présentée plus en détail dans les sections qui suivent. Ensuite, nous présenterons un ensemble de défis reliés à leur réalisation ou à leur utilisation.

\section{Illustration : le portail de l'automobile COVISINT}

COVISINT est né en février 2000 sur l'initiative des trois géants de l'automobile américains soit, Ford Motors, General Motors et le groupe DaimlerChrysler. Le projet consiste à construire un portail public pour l'industrie automobile. Le portail doit consister en une plate-forme d'achat, un espace de travail virtuel et un système de support à la chaîne d'approvisionnement. L'objectif fixé est de réduire de 3,000 \$US les coûts de productions d'un véhicule de 19,000 \$US ainsi que de réduire le temps du cycle de développement d'un véhicule de 42 mois à aussi peu que de 12 à 18 mois. COVISINT doit rassembler 90,000 entreprises pour un volume transactionnel espéré avoisinant les 240 milliards de dollars par année. II s'agit sans aucun doute du projet le plus ambitieux en ce moment en terme de développement de système d'informations interorganisationnels et qui a la particularité intéressante d'intégrer les trois formes d'interactions présentées dans ce rapport.

En terme de SIIO d'information partagée, COVISINT est avant toute autre chose une place de marché électronique qui espère réduire grandement les coûts de transaction pour les approvisionnements. La place de marché possède un mécanisme de prix statique mais également un mécanisme de négociation permettant de réaliser des enchères inversées ("request for quote") à partir d'un catalo- 
gue unifié pour l'ensemble des utilisateurs. Le catalogue de produits peut également être personnalisé par organisation. Au-delà de cette fonction d'achat de pièce, la place de marché sert également de communauté virtuelle pour la gestion, l'achat ou la vente d'actifs spécifiques inutilisés. COVISINT offre également des SIIO de support à la chaîne d'approvisionnement qui gèrent les niveaux d'inventaire, fournissent des estimations de production, détaillent l'état des commandes entre deux partenaires sur la place de marché. Un service d'extranet est également présent de façon à partager avec ses sous-traitants les informations qu'un manufacturier souhaiterait transmettre telles que des cédules ou d'autres détails de production.

Enfin, COVISINT se veut un espace de travail en collaboration inter-organisationnel qui permet le stockage centralisé de documents, offre un espace virtuel de conception assistée par ordinateur, des outils de gestion de projet et d'autres outils de collaboration.

De plus, la place de marché offre des services pour faciliter l'intégration de ses systèmes aux systèmes de gestion intégrés des partenaires, mais également des services financiers pour le financement d'achats réalisés sur la place. Enfin, le portail se veut une communauté virtuelle pour l'industrie automobile qui permet de fournir des nouvelles sur l'état général du marché.

Toujours en cours de développement, la place de marché avait géré à la mi-août 2001 un volume transactionnel de 129 milliards de dollars américains depuis le début de l'année, ce qui poussait déjà de nombreux analystes à considérer le portail comme un succès. Néanmoins, de nombreux défis restent à être relevés car plusieurs constructeurs refusent toujours de joindre la place de marché, la trouvant inadaptée à leur chaîne d'approvisionnement et étant réticents de partager trop d'informations sur le portail. De plus, plusieurs doutent que l'on puisse fluidifier les processus interorganisationnels dans l'industrie automobile aussi facilement que dans le cas de DELL ou même réaliser un environnement de travail en collaboration dans un secteur industriel où la concurrence est assez féroce.

Néanmoins, les premiers chiffres réalisés méritent qu'on s'intéresse à ce modèle de portail industriel gérant une grande variété de SIIO car le succès de COVISINT impliquerait sans aucun doute que le modèle soit appliqué à d'autres industries. 


\section{Information partagée : Les hubs électroniques et les places de marché}

Grâce à Internet, vendeurs et acheteurs interagissent de plus en plus sur les places de marché électroniques pour :

- Identifier des partenaires d'affaires potentiels,

- Sélectionner un partenaire d'affaires

- Exécuter une transaction
Les systèmes d'information partagée forment la première catégorie de systèmes inter-organisationnels. Ils permettent à différentes organisations d'échanger de l'information de manière centralisée.

\subsection{Les places de marché : " an extranet on steroids ?"}

Les places de marché se définissent comme un système d'information interorganisationnel où de multiples acheteurs et vendeurs interagissent pour accomplir une des activités de marché suivantes : (1) identifier des partenaires d'affaires potentiels, (2) sélectionner un partenaire d'affaires potentiel et (3) exécuter une transaction $^{3}$. Il ne s'agit en rien d'un concept nouveau puisque les premières places de marché remontent aux années 70 avec les premiers systèmes de réservation de billets d'avion (le fameux système SABRE) et les premiers systèmes de courtage électroniques. Néanmoins, jusqu'à très récemment, la conception d'un tel système nécessitait l'utilisation d'applications propriétaires. Aujourd'hui, l'utilisation d'Internet et en particulier du web a permis de généraliser grandement l'utilisation de ces systèmes.

\subsection{Fonctions et caractéristiques}

Une place de marché possède 5 fonctions.

(1) une fonction de rencontre : Une place de marché doit permettre de faire un match entre un acheteur et un vendeur. Ce contact est fait en fonction de différents critères tels que le produit offert ou demandé, ses moyens financiers, sa situation géographique etc.

(2) une fonction de catalogage : Une place de marché centralise le catalogage des produits vendus par les différents membres de la place réduisant ainsi les asymétries d'information.

(3) une fonction de négociation : Un mécanisme de négociation doit permettre d'exécuter une transaction sur une place de marché. Ces mécanismes peuvent être très divers et être statiques (prix fixes) pour des produits très spé-

\footnotetext{
3 Traduction libre de Choudury et al., "Uses and Consequences of Electronic Markets: An Empirical Investigation in the Aircraft Parts Industry”, MIS Quarterly, Dec. 1998, 471-507.
} 
cifiques ou dynamiques (mécanismes d'enchères) pour des biens plus homogènes.

(4) une fonction logistique : Une place de marché se doit généralement de fournir des services connexes à une transaction réalisée sur son site. Ces services peuvent être très restreints et consister simplement en l'envoi de confirmation et de facturation ou être beaucoup plus complexes et consister en des services de livraison ou de financement.

(5) un cadre légal et institutionnel : Une place de marché offre généralement un cadre légal d'échange. En effet, effectuer des transactions sur un tel site implique généralement de respecter des critères au niveau des délais de paiement, de livraison etc. À un niveau plus avancé, la place de marché peut devenir une véritable communauté virtuelle diffusant des informations sur l'industrie, en offrant des groupes de discussions ou d'autres services.

Une place de marché peut être neutre ou biaisée. Une place neutre est gérée par un opérateur indépendant alors qu'une place dite biaisée est gérée par une ou plusieurs des organisations qui utilisent la place. Généralement, cette deuxième situation implique d'avoir un pouvoir de marché relativement important sans toutefois être trop dominant de façon à éviter une situation concurrentielle illégale.

Le nombre de formes de places de marché est encore vaste aujourd'hui. Selon le Gartner Group (2000) ${ }^{4}$, seules trois formes de places de marché devraient se distinguer d'ici à 2005 :

- La première forme est celle des places de marché de commodités qui se spécialisent dans la vente de biens homogènes et plus particulièrement des matières premières tel que l'acier, les plastiques ou les produits chimiques. L'existence de ces places est justifiée par l'importance des gains espérés par l'agrégation du marché en un même lieu virtuel de façon à supprimer un trop grand nombre d'intermédiaires présents dans la chaîne d'approvisionnement et aussi de façon à profiter de mécanismes transactionnels efficaces par enchères. La place de marché e-steel dans le domaine de l'acier en est un exemple.

\footnotetext{
4 "Gartner Says Three E-Marketplace Models Will Control the B2B Market by 2005", The Gartner Group, 2000
}

\section{Les cinq fonctions d'une} place de marché

- Fonction de rencontre

- Fonction de catalogage

- Fonction de négociation

- Fonction logistique

- Cadre légal et institutionnel

Les places de marché de commodités entraînent des gains espérés importants en réduisant le nombre d'intermédiaires et en utilisant des mécanismes transactionnels efficaces. 
- La seconde forme est la place de marché de services qui a pour fonction de supporter des processus particuliers d'une organisation tels que ceux de livraison, de gestion des excédents d'actifs ou reliés au financement. Ces places de marché peuvent également servir

La place de marché de services supporte des processus particuliers d'une organisation tels que ceux de livraison ou de gestion, tandis qu'une place de marché d'intégration servira d'infomédiaire entre de nombreuses organisations.

Décider de ne pas joindre une place de marché équivaut à abandonner à la concurrence tout un ensemble de contrats potentiels. à compléter des transactions effectuées sur d'autres places de marché. La place de marché Virtual Chip Exchange, une coentreprise à laquelle participe notamment la firme québécoise Mediagrif, se spécialise par exemple dans l'écoulement de stocks de composants électroniques excédentaires.

- La troisième est celle des places de marché d'intégration dont le rôle sera d'être un infomédiaire destiné à faciliter l'intégration des processus (et donc des systèmes d'information) entre de nombreuses organisations.

Il est important de noter que ces formes ne sont pas mutuellement indépendantes et que certains portails industriels (tels que COVISINT dans le secteur de l'automobile) combinent les trois formes citées plus haut. Ces portails sont un carrefour d'information permettant aux membres d'avoir accès à ces différents services via un site centralisé.

\subsection{Joindre ou ne pas joindre une place de marché?}

La décision de joindre une place de marché peut sembler attirante à première vue en raison des économies que l'on espère pouvoir y faire. Néanmoins, beaucoup de facteurs sont à prendre en compte lorsque l'on décide de joindre une place de marché ou non.

Premièrement, la question la plus fondamentale consiste à se demander si notre organisation peut se permettre de ne pas utiliser un tel système. Pour certains soustraitants, qui dépendent seulement d'un ou deux manufacturiers, la décision de joindre le système se fait sous contrainte. De plus, plusieurs organisations gouvernementales ont décidé de ne considérer comme fournisseurs potentiels que ceux qui utilisent leurs portails pour transiger avec eux (notamment pour transmettre les soumissions en réponse aux appels d'offres). Décider de ne pas utiliser un tel système équivaut à abandonner à la concurrence tout un ensemble de contrats potentiels. 
Ensuite, il est important d'évaluer à quel point la place peut s'intégrer facilement à nos processus et à nos systèmes actuels. Il faut que le volume d'affaires généré par la place de marché couvre au moins les frais d'accès et d'adaptation associés à son utilisation. L'organisation pourrait avoir à investir tant en matériel, en logiciel, qu'en expertise technique pour pouvoir utiliser la place de marché électronique.

Finalement, il est important de ne pas s'aliéner des distributeurs ou des grossistes avec lesquels une relation d'affaires efficace est en cours et qui sont la source d'un avantage stratégique. Si un client a une relation à long terme avec des fournisseurs et que cette relation lui donne beaucoup de flexibilité, il tentera de conserver cette relation plutôt que d'essayer de sauver quelques dollars en utilisant un site mettant en compétition une multitude de fournisseurs.
Page 15

La place de marché ne doit pas menacer les relations d'affaires existantes. De plus, le volume d'affaires généré par la place de marché doit couvrir les frais d'accès et d'adaptation associés à son utilisation.

\section{Quelques questions clés à se poser avant de joindre une place de marché}

- Est-ce que la structure de la place de marché me favorise (ou me défavorise)?

- Est-ce que j'ai les systèmes pour accéder facilement à la place de marché?

- Est-ce que les coûts d'accès sont raisonnables?

- Est-ce que cet accès s'intègre bien à mes processus d'affaires?

- Est-ce que cette place de marché menace des relations d'affaires existantes?

- Est-ce que mes partenaires d'affaires migrent vers les places de marché?

- Est-ce que ces places me donnent accès à de nouveaux marchés? 


\section{Interdépendance séquentielle: systèmes d'information inter-organisationnels et chaîne d'approvisionnement}

Alors que la chaîne de valeur correspond à la séquence des activités ajoutant de la valeur aux produits ou services à l'intérieur d'une entreprise, la chaîne d'approvisionnement correspond à une relation séquentielle de chaînes de valeurs de plusieurs entreprises.
La seconde forme de système inter-organisationnel est l'interdépendance séquentielle, où les systèmes sont reliés de manière cohérente avec la séquence d'activités de la chaîne d'approvisionnement.

\subsection{Concepts de chaîne d'approvisionnement}

Il est d'abord important de différencier les concepts de chaîne d'approvisionnement et de chaîne de valeur. En effet, la chaîne de valeur correspond à la séquence des activités à l'intérieur d'une entreprise qui vont ajouter de la valeur aux produits et/ ou services que l'organisation vend à ses clients et pour lesquels elle reçoit un paiement.

La chaîne d'approvisionnement, quand à elle, se situe à un niveau plus macro et correspond à une relation séquentielle de chaînes de valeurs de plusieurs entreprises, c'est-à-dire l'ensemble des processus allant de l'achat de la matière première initiale jusqu'à la consommation du produit fini et ce dans une relation séquentielle soustraitants/acheteurs.

Il s'agit donc des fonctions à l'intérieur et à l'extérieur d'une entreprise qui permettent de fabriquer des produits et/ou d'apporter un service au consommateur. Une chaîne d'approvisionnement dans sa plus simple expression est illustrée par la figure 3 .

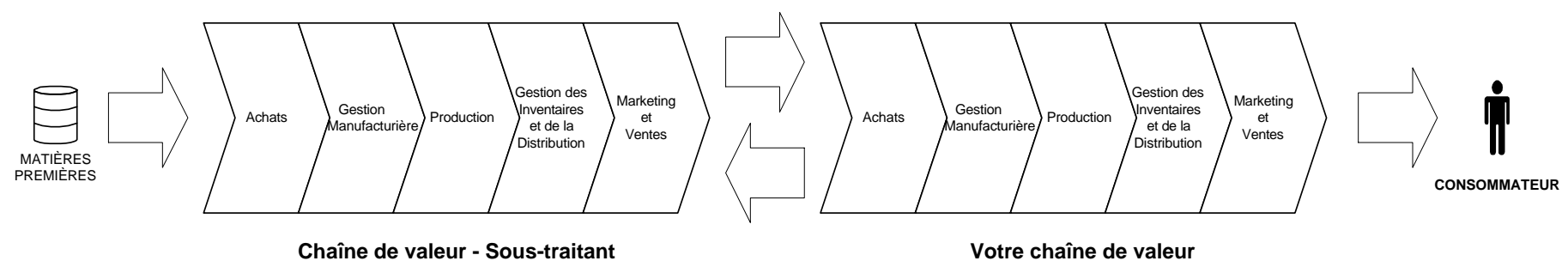

Figure 3 - Chaîne d'approvisionnement simple 
Cette chaîne peut aisément se compliquer si l'organisation fait appel à plusieurs niveaux de sous-traitance ou si elle réalise un produit qui commande d'autres transformations. On peut alors se retrouver dans une situation correspondant à la figure 4. Dans ce cas précis, on fait affaire avec de multiples niveaux de sous-traitance qui impliquent autant de relations à gérer entre organisations.

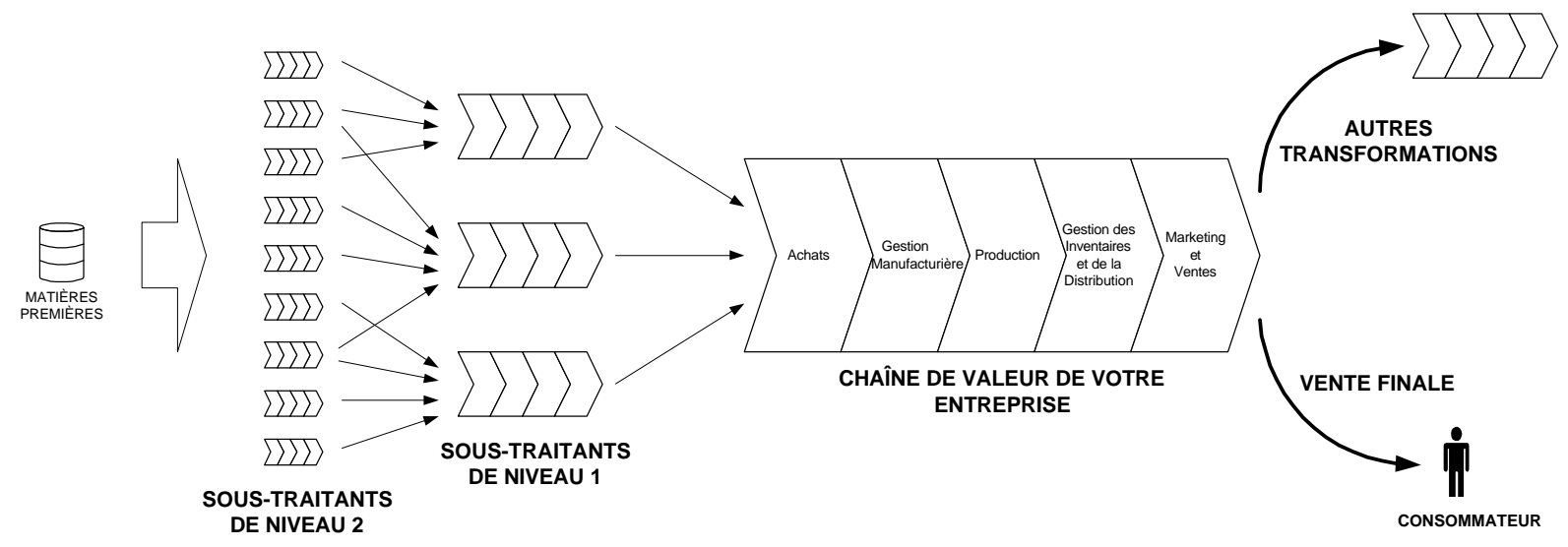

Figure 4 - Chaîne d'approvisionnement complexe

Une gestion efficace de la chaîne d'approvisionnement est présentée depuis quelques années comme étant une façon d'améliorer l'efficacité organisationnelle en général et également de dégager un avantage concurrentiel. Pour ce faire, on vise plusieurs objectifs parmi lesquels se trouvent la réduction du temps du cycle, la réduction des inventaires, une plus grande rapidité d'adaptation aux changements, une meilleure efficacité des processus d'affaires et de leur intégration. Tous ces objectifs consistent à avoir ultimement un consommateur plus satisfait du produit ou service qu'il aura acquis.

On peut définir la gestion de la chaîne d'approvisionnement selon deux perspecti$\operatorname{ves}^{5}$ : celles des biens et de l'information correspondante, ou celle des processus d'affaires. La première perspective est la suivante :

La gestion de la chaîne d'approvisionnement consiste en la gestion de l'ensemble des activités associées (1) aux flux et à la transformation

\footnotetext{
${ }^{5}$ Premkumar, Prem ; Information System Management, summer 2000, pp.56-59
}

Une gestion efficace de la chaîne d'approvisionnement vise, entre autres, la réduction du temps du cycle, la réduction des inventaires et une plus grande rapidité d'adaptation aux changements. 
des biens, allant de la matière première jusqu'à l'utilisateur final, et (2) aux flux d'information correspondant obtenus par des relations d'approvisionnement améliorées de façon à en dégager un avantage compétitif ${ }^{6}$.

La figure 5 correspond à cette définition. On y présente une chaîne d'approvisionnement typique allant du fournisseur de matières premières jusqu'au consommateur. De façon générale, les biens se déplacent vers la droite, c'est à dire du fournisseur

Deux visions de la chaîne d'approvisionnement :

\section{- Gestion des activités}

associées aux flux et à la transformation des biens ainsi qu'aux flux d'information

\section{- Intégration des processus} d'affaires, allant du premier fournisseur au consommateur final. jusqu'au consommateur, les paiements allant logiquement dans la direction inverse. L'information, quant à elle, circule dans les deux sens. Elle peut aller dans le sens de la chaîne (dans le cas d'une facture) ou dans le sens contraire (dans le cas d'un bon de commande ou de spécifications techniques par exemple).

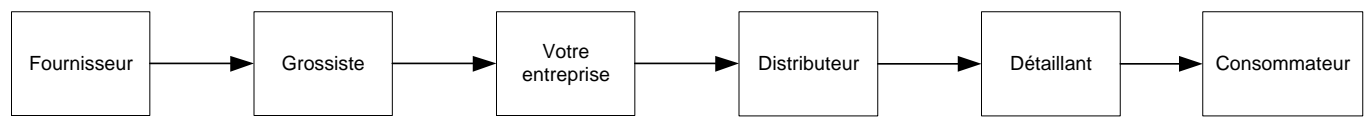

Biens physiques

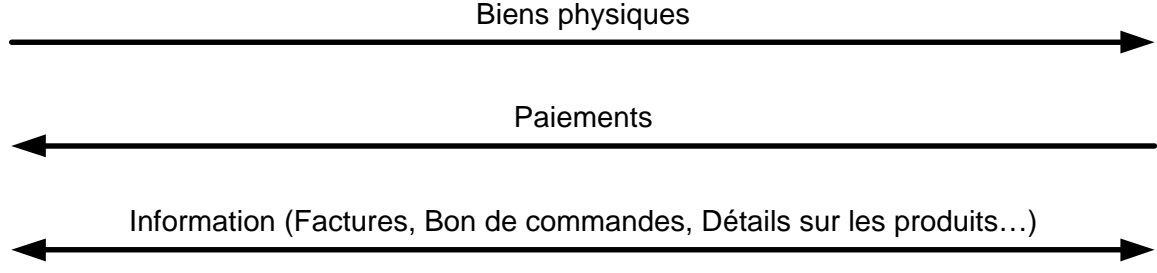

Figure 5 - Chaîne d'approvisionnement et flux correspondants ${ }^{7}$

L'autre perspective de définition de la gestion de la chaîne d'approvisionnement est la suivante :

La gestion de la chaîne d'approvisionnement correspond à l'intégration des processus d'affaires, allant du premier fournisseur au consommateur final, qui apportent des produits, des services et de l'information et qui ajoutent de la valeur pour le consommateur.

${ }^{6}$ Traduction libre et adaptée de Handfeld et Nichols, 1999, Introduction to Supply Chain Management, Prentice Hall, Englewood cliffs, NJ.

${ }^{7}$ Adapté de : Premkumar, Prem ; Information System Management, été 2000, pp.56-59 
La figure 6 présente cette vision de la chaîne d'approvisionnement où chaque étape de la chaîne de valeur correspond à un ensemble de processus d'affaires. L'intégration entre organisation se fait par échange d'information ou de biens. Ainsi, le processus d'achat d'une organisation est intégré à celui de la planification chez son fournisseur lorsqu'un bon de commande lui est transmis. Il en est de même lors de la livraison d'un bien.

Néanmoins, il est important de noter que l'intégration des processus d'affaires se fait à de multiples niveaux qui vont bien au-delà des documents transactionnels et des biens livrés. Il suffit de penser à l'envoi de schémas techniques, à de la planification et à de la gestion de projets en commun, à l'échange de documents de contrôle de qualité, à de la vérification de niveaux d'inventaires etc. À cela, il faut ajouter toutes les communications se faisant de façon moins formelle.

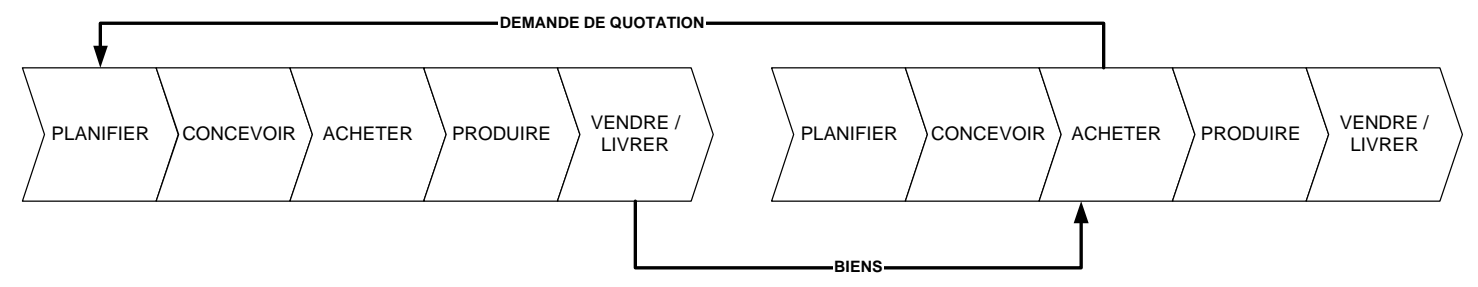

L'intégration des processus d'affaires se fait à de multiples niveaux qui vont au-delà des documents transactionnels et des biens livrés.

Figure 6 - Intégration de chaînes de valeur par flux d'information et de biens

Dans la seconde partie de ce chapitre, nous allons présenter des technologies permettant de supporter les activités reliées à ces flux inter-entreprises.

\subsection{Systèmes d'information en support à la chaîne de valeur ou à la chaîne d'approvisionnement}

Beaucoup d'organisations possèdent maintenant des systèmes d'information internes qui permettent de soutenir les processus de gestion de leur chaîne de valeur mais ils consistent rarement en des outils d'intégration complexes avec leurs différents partenaires commerciaux. Nous allons ici présenter des outils d'intégration dans le cadre d'une relation de chaîne de valeur. 
Deux critères de sélection pour un SIIO :

- le mode d'accès à l'information d'une organisation à l'autre

- le type d'accès permis par le système
Les flux d'information entre organisations peuvent se dérouler de deux façons : soit directement d'une organisation à l'autre soit au travers d'un intermédiaire qui concentre les flux d'information. La plupart des relations inter-organisationelles se font aujourd'hui de façon directe mais les dernières années ont vu l'essor du modèle de place de marché électronique. Ces importants systèmes inter-organisationnels concentrent généralement dans un même secteur industriel un grand nombre de transactions se déroulant à plusieurs niveaux de la chaîne d'approvisionnement. Les technologies que nous allons présenter ici peuvent être utilisées dans les deux modèles.

On devra considérer deux critères de sélection pour un système interorganisationnel : le mode d'accès à l'information d'une organisation à l'autre et le type d'accès permis par le système. Le mode d'accès peut être basé par un échange de messages simples entre organisations ou peut être de nature beaucoup plus interactive. Le type d'accès permis peut consister en une simple consultation d'information. Il peut éventuellement permettre des mises à jour ou des modifications dans les bases de données d'une autre organisation.

Comme le montre la figure 7 , nous avons retenu quatre technologies interorganisationnelles soit l'EDI, XML, les Extranets ainsi que les applications propriétaires qui sont classées en fonction des critères que nous venons de définir. Il ne s'agit pas de technologies mutuellement exclusives puisqu'elles peuvent être utilisées en combinaison. De plus, elles se recoupent sur bien des niveaux. Nous avons classé chacune de ces technologies dans la figure 5 en fonction des deux critères que nous venons tout juste de définir.

On peut remarquer que ces technologies se situent sur un axe en diagonale qui correspond naturellement à une architecture d'intégration inter-organisationelle allant de très faiblement couplée pour l'EDI à parfaitement intégrée pour les applications propriétaires. Nous allons faire la présentation de ces quatre technologies et tenter de déterminer dans quelles conditions elles peuvent soutenir les activités de la chaîne d'approvisionnement d'une industrie. 


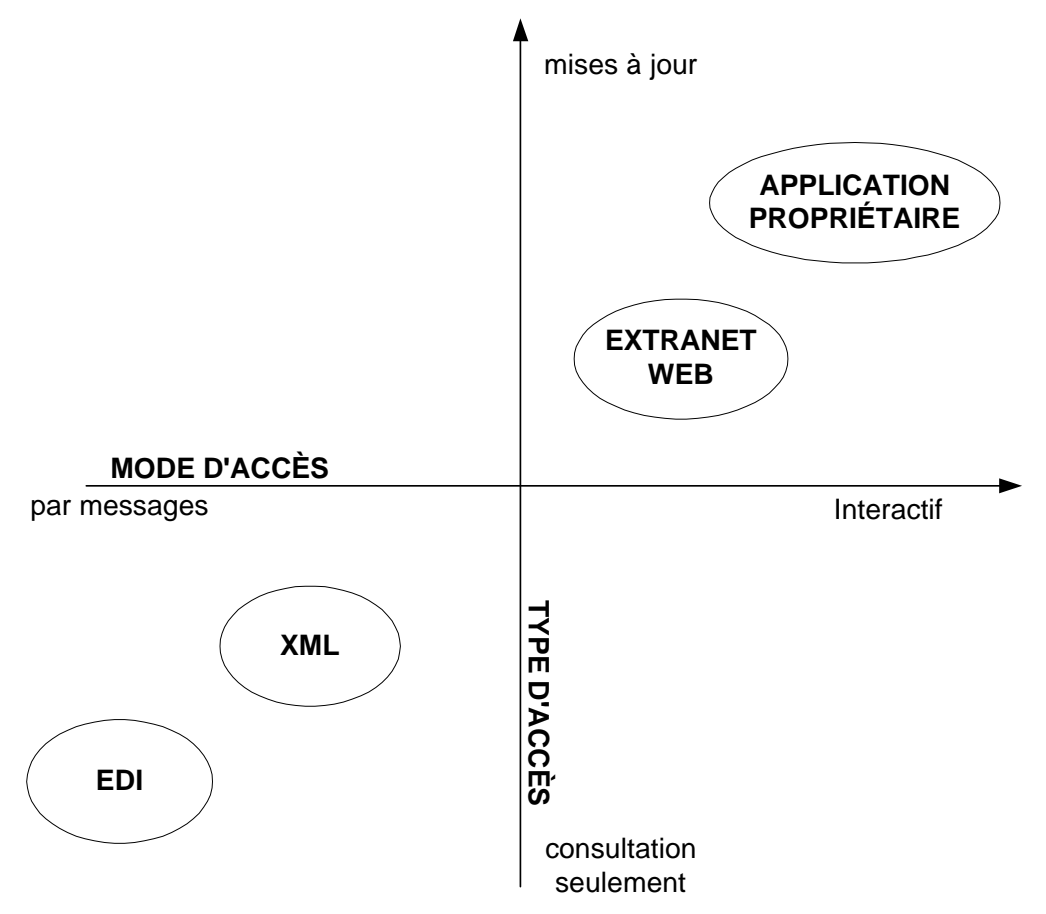

Figure 7 - Classification des technologies en support à chaîne de valeur

Chaque technologie est présentée brièvement dans les pages qui suivent.

\section{- $\quad$ EDI}

L'EDI est une technologie vieille de près de 30 ans. Néanmoins, son essor et la généralisation de son utilisation sont encore très récents. On définit cette technologie comme suit :

\section{L'EDI correspond au transfert d'information d'un système à un autre dans un format structuré et prédéterminé.}

En fait, il s'agit de la technologie pionnière dans le domaine du commerce électronique, car bien avant l'internet ou les premiers systèmes inter-organisationnels interactifs, des organisations s'échangeaient déjà des documents électroniquement par cette méthode. Les premiers documents échangés entre systèmes correspondaient aux documents d'affaires les plus classiques tels que des bons de commande ou des factures. Mais avec le temps, l'EDI est venu couvrir des documents plus variés tels que des spécifications techniques, des catalogues, des confirmations, ou même des messages textes.
L'EDI, technologie pionnière dans le domaine du commerce électronique, correspond au transfert d'information d'un système à un autre dans un format structuré et prédéterminé. 
L'EDI fonctionne par échange de messages, ou ensemble de transactions, entre systèmes. Les systèmes EDI originaux étaient des systèmes destinés aux achats et sont encore les plus répandus aujourd'hui. La figure 8 illustre un tel système. Par un tel système, on cherche à éliminer les commandes réalisées par courrier ou par fax pour les remplacer par des transactions électroniques pouvant éventuellement être auto-

Avec un système EDI, on cherche à remplacer les commandes réalisées par courrier ou par fax par des transactions électroniques pouvant éventuellement être automatisées. matisées lorsque l'inventaire est trop bas. La transmission des messages peut se faire sur un réseau privé ou public tel qu'Internet. Les réseaux privés, souvent appelés des Réseaux à Valeur Ajoutée (RVA), sont généralement gérés par des firmes de télécommunications et fournissent les services de connexions entre les deux partenaires, voire également des services professionnels en terme de services et de matériels reliés à l'EDI.

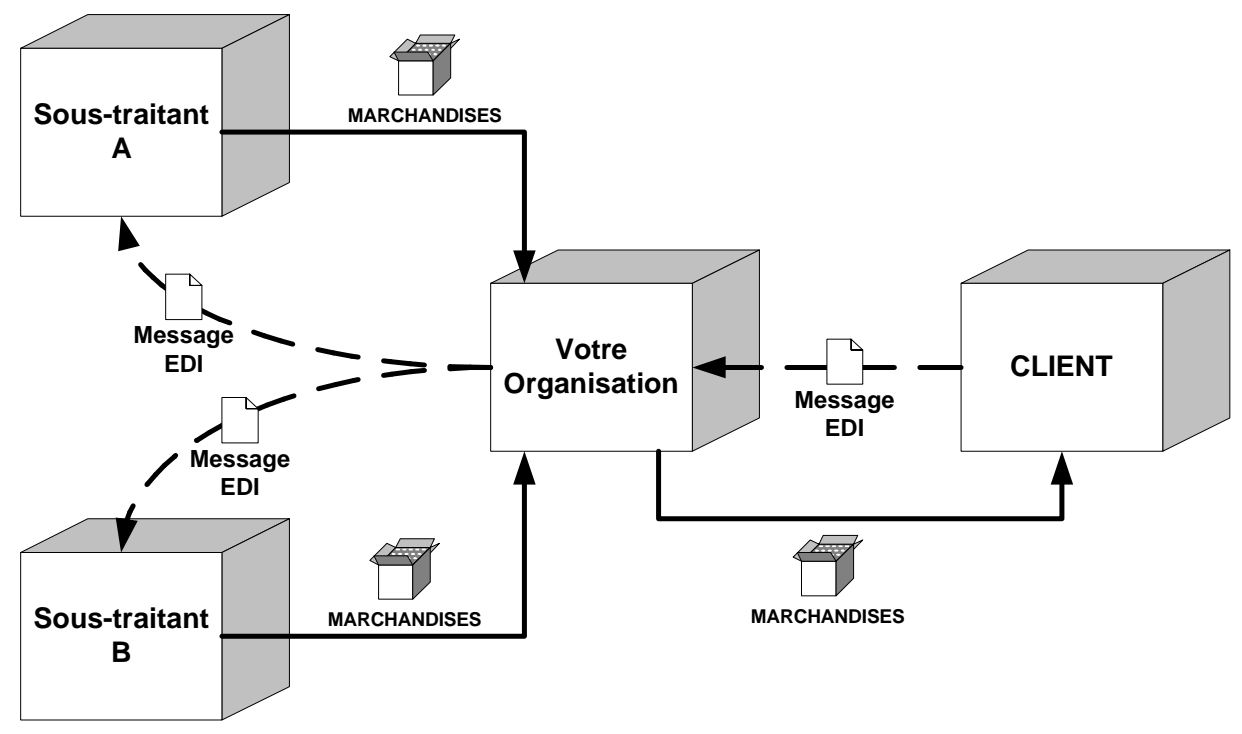

Figure 8 - EDI et commerce électronique B2B

Ainsi, si un système EDI peut paraître simple conceptuellement, il s'agit en réalité d'une technologie qui présente une grande complexité pour être implantée, et ce, particulièrement en raison de la structure qui est utilisée pour le formatage des messages échangés (pour lequel il existe deux standards dominants). Composé de codes complexes, un message est généralement impossible à déchiffrer à sa simple lecture et cette complexité rend plus difficile, voire impossible, la création d'un niveau d'interactivité avancé entre deux partenaires d'affaires. De plus, de tels systèmes 
impliquent généralement des investissements importants pour les intégrer aux systèmes hérités internes, et ce, autant au niveau de l'envoi que de la réception des messages. Ces raisons expliquent pourquoi, bien qu'existant depuis plus de 30 ans, l'EDI n'a pas généré un accroissement majeur du commerce électronique B2B. Ainsi, les systèmes existants à ce jour sont généralement limités à des messages transactionnels (factures ou bons de commandes) et à des relations entre manufacturier et sous-traitant ou fournisseurs de premier niveau.

\section{Néanmoins, il est important de noter que l'EDI peut encore parfaitement être} une technologie avantageuse à ce jour. En fait, la technologie EDI ne semble pas destinée à disparaître, notamment en raison de l'essor de l'utilisation du web comme réseau de transmission permettant désormais à des plus petites firmes de s'offrir l'EDI comme façon de se relier à ses partenaires d'affaires sans devoir payer les coûts importants reliés à l'utilisation d'un RVA.

En fait, deux questions devront être posées si l'on envisage l'implantation d'un système EDI :

- Est-ce que le système EDI devra être intégré à de nombreux systèmes différents à l'interne ainsi qu'à l'externe ?

- Avons-nous besoin d'un grand niveau d'interactivité dans notre relation d'affaires ?

Si la réponse aux deux questions s'avère être non, ce qui pourrait s'illustrer par une situation où un manufacturier équipé d'un système intégré de type ERP souhaite se relier à ses quelques sous-traitants équipés eux aussi d'un système ERP et qu'en plus, on ne souhaite qu'échanger des bons de commandes et des factures, alors l'EDI sera la technologie à retenir.

Néanmoins, si l'on souhaite un niveau d'interactivité plus grand entre partenaires d'affaires en échangeant par exemple des documents plus complexes ou si l'on désire des possibilités avancées de dialogues entre systèmes, des alternatives telles que XML seront à considérer .

\section{En dépit de la complexité de langage et l'existence de standards différents, la technologie EDI peut se révéler avantageuse. Des petites firmes peuvent désormais se relier à leurs partenaires d'affaires avec EDI, sans devoir payer les coûts importants d'utilisation d'un RVA.}

Si l'on souhaite un niveau élevé d'interactivité entre partenaires d'affaires ou si l'on désire des possibilités avancées de dialogues entre systèmes, on devra considérer des alternatives à l'EDI. 


\section{- XML}

Certaines difficultés sont associées à l'EDI, notamment en raison de la complexité du langage et de l'existence de standards différents. Il existe aujourd'hui d'autres langages de définition documentaire. Le plus célèbre est sans aucuns doutes XML (eXtensible Markup Language). Nous n'entrerons pas dans des explications de nature trop technique mais l'essor important prévu pour cette technologie nécessite de s'y attarder.

XML est un langage pour structurer des données sous un format texte. On peut structurer sous un format XML une facture, des spécifications techniques, des transactions financières ou même des CVs. En fait, à peu près n'importe quel document d'affaires peut être structuré au format XML. XML définit un ensemble de règles de formatage et de structure pour le document de façon à ce qu'il soit facile à générer et à interpréter par différents systèmes (et technologies), qu'il ne soit pas ambigu et enfin qu'il soit extensible si l'on souhaite lui ajouter de l'information.

Un important avantage associé à XML est d'être utilisable sur le web. En effet, l'intégration entre deux systèmes avec XML peut se faire de deux façons, tel qu'illustré à la figure 9. C'est-à-dire soit par échange de documents ou de messages (d'une manière similaire à l'EDI) soit par intégration d'applications par le web. Dans ce deuxième cas, on parle alors de web services.

Intégration par messages XML

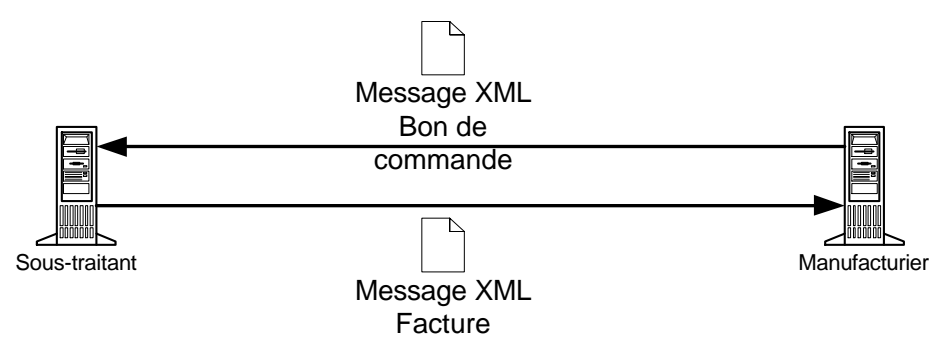

Intégration d'applications: HTTP+XML (Web Services)

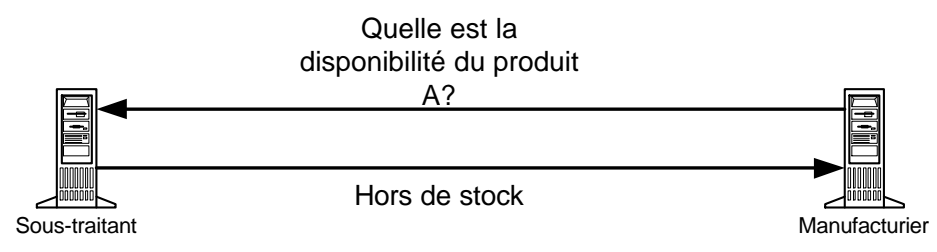

Figure 9 - Méthodes d'intégration B2B 
Les services web sont des applications qui sont appelées par le web (via http) en utilisant un ensemble de standards tels que SOAP et XML. SOAP est un protocole au format XML qui permet d'appeler des services web indépendamment du type de plate-forme utilisée et qui utilise le protocle http du web pour le transport et XML pour les données. L'intégration peut alors devenir beaucoup plus complexe et ne se limite alors plus à un simple échange documentaire mais à un véritable appel d'applications à distance pour obtenir les informations souhaitées. Un tel système permet donc un dialogue plus riche entre partenaires d'affaires et donc un support à des processus d'affaires inter-organisationnels plus complexes.

\section{- Extranets}

Tel qu'illustré auparavant à la figure 7, un extranet permet un mode d'accès beaucoup plus interactif. En fait, il s'agit d'une version d'un intranet corporatif mais destinée à des utilisateurs externes à une organisation. Cette utilisation externe est généralement destinée aux fournisseurs, distributeurs ou clients d'une organisation. La différence entre l'EDI et cette technologie se situe essentiellement au niveau de l'interactivité possible entre les deux organisations. Alors que l'EDI utilise une architecture faiblement couplée par transmission de messages au format propriétaire, l'extranet permet d'accéder à des informations beaucoup plus variées au travers de l'internet. Ainsi, dans une relation de chaîne d'approvisionnement telle qu'illustrée sur la figure 10, l'extranet permet de supporter plusieurs niveaux de la chaîne de valeur et surtout une bien plus grande variété d'informations.

On peut ainsi vérifier le niveau des stocks de nos clients, aller chercher des spécifications techniques sur des pièces à produire ou vérifier l'état d'une commande, alors que l'EDI se limite bien souvent à des envois unidirectionnels de bons de commande. Naturellement, l'accès à ce type d'information requiert un niveau de confiance élevé avec ses partenaires industriels.
L'extranet permet d'accéder à des informations variées via

Internet. On peut ainsi vérifier le niveau des stocks des clients, aller chercher des spécifications techniques sur des pièces à produire ou vérifier l'état d'une commande. 


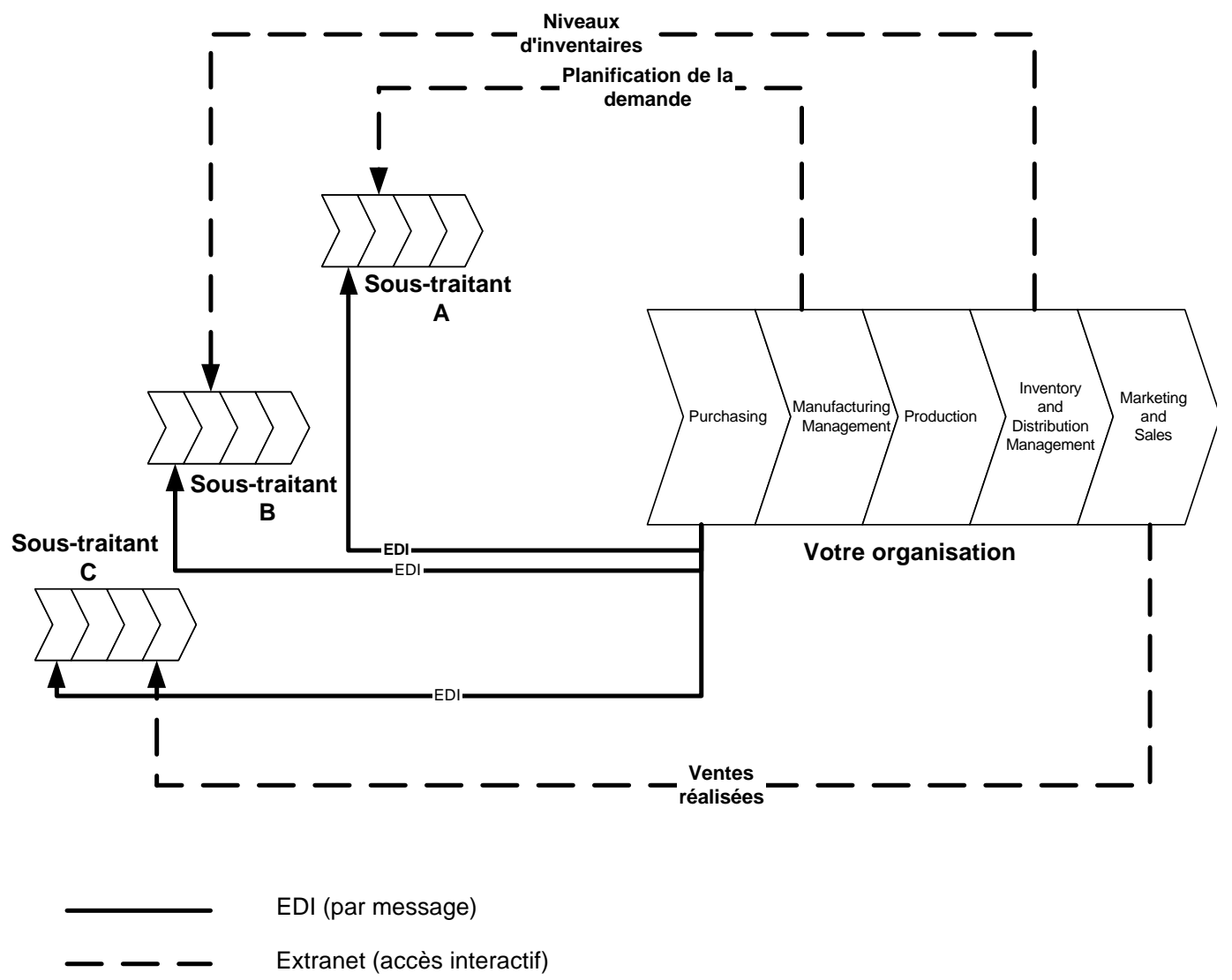

Figure 10 - Un extranet dans une relation de chaîne de valeur

La mise en place d'un extranet est relativement simple. Elle nécessite cependant l'installation d'un firewall afin de protéger les systèmes internes contre des intrusions intempestives.
D'un point de vue technologique, la mise en place d'un extranet se veut relativement simple. La figure 11 présente une architecture possible. Elle consiste en la mise en place d'un serveur Web et de liens avec les applications de l'intranet. Il est par contre nécessaire de protéger les systèmes internes par la mise en place d'un firewall (pour empêcher les intrusions dans le système par des personnes non autorisées).

L'utilisation d'un extranet présente plusieurs avantages. Le premier avantage est que l'accès est possible à partir de n'importe quelle plateforme permettant d'accéder au web. Pour un sous-traitant de bas niveau qui ne peut se permettre des investissement importants en EDI, l'extranet permet d'accéder, à un très faible coût, à des informations venant de son manufacturier de façon électronique. Un autre avantage se situe au niveau de la plus grande interactivité. Par exemple, certains manufacturiers passent leurs commandes par EDI mais donnent accès aux spécifications à suivre par un extranet corporatif pour profiter de la plus grande souplesse d'utilisation. On peut ainsi partager des informations plus variées et notamment des chiffres de planifications permettant ainsi d'adapter la production et donc les niveaux d'inventaire. 
La relation client-fournisseur devient alors plus étroite et généralement plus efficace.

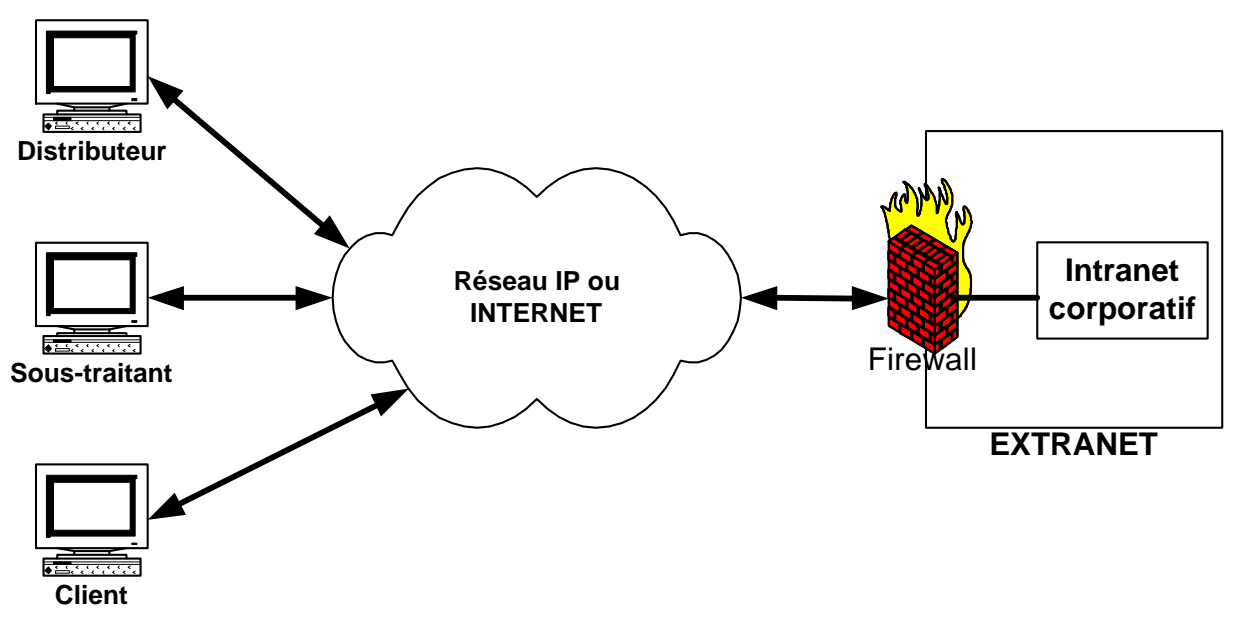

Figure 11 - Architecture possible pour un extranet

Les défauts à retenir d'un tel système sont tout d'abord techniques et se situent au niveau de l'intégration avec les systèmes hérités qui n'ont généralement pas été conçus pour transmettre de l'information sur le web. Dans certains cas, l'intégration peut devenir coûteuse. Une autre problématique se situe au niveau de la sécurité. Nous avons vu que l'architecture utilisée impliquait généralement une transmission de l'information par le web risquant ainsi d'être éventuellement interceptée.

\section{- Applications propriétaires}

La dernière technologie inter-organisationelle dont nous allons traiter consiste en une application propriétaire. Un exemple de ces technologies est le système de réservation SABRE qui utilise une application propriétaire chez les agences de voyage. Elle consiste généralement en une architecture client/serveur. Ces applications sont maintenant parfois complémentaires avec des applications web.

En fait, la différence est maintenant mince entre les deux technologies. À ce jour, on maintient un niveau d'interactivité et de performance supérieur par rapport à un extranet Web lorsqu'on utilise une application propriétaire. Qui plus est, le niveau de sécurité est plus élevé puisqu'on n'utilise pas le réseau Internet. Néanmoins, une mise à jour logicielle implique la réinstallation des applications chez tous les parte-

\section{Qualités de l'extranet}

- Possibilité d'accès à partir de toute plateforme

- Plus grande interactivité

Défaut de l'extranet

- Difficulté d'intégration avec les systèmes hérités

- Problématique au niveau de la sécurité 
naires. Généralement, on réservera l'utilisation de cette technologie lorsque des niveaux d'interactivité élevés sont nécessaires. Ce mode correspond rarement à une relation de chaîne d'approvisionnement. Il est plutôt limité à la transmission de do-

Une application propriétaire permet d'obtenir un niveau élevé de sécurité et d'interactivité. cuments d'affaires.

\subsection{Synthèse des technologies}

Le tableau suivant résume bien les éléments clefs associés à chaque technologie.

\begin{tabular}{|c|c|c|c|c|}
\hline & EDI & XML & EXTRANET & $\begin{array}{l}\text { APPLICATION } \\
\text { PROPRIÉTAIRE }\end{array}$ \\
\hline Processus supportés & $\begin{array}{l}\text { Support à des processus } \\
\text { d'affaires inter- } \\
\text { organisationnels formels } \\
\text { et simples }\end{array}$ & $\begin{array}{l}\text { Support à des processus } \\
\text { d'affaires inter- } \\
\text { organisationnel formels et } \\
\text { complexes }\end{array}$ & $\begin{array}{l}\text { Support à des processus } \\
\text { d'affaires inter- } \\
\text { organisationnels ad hoc }\end{array}$ & $\begin{array}{l}\text { Support à des processus } \\
\text { d'affaires inter- } \\
\text { organisationnels ad hoc }\end{array}$ \\
\hline $\begin{array}{l}\text { Environnements } \\
\text { technologiques } \\
\text { (internes et externes) }\end{array}$ & -homogènes & -hétérogènes & -accès Web & $\begin{array}{l}\text {-nécessite un accès à un } \\
\text { réseau privé }\end{array}$ \\
\hline Avantages & $\begin{array}{l}\text {-Technologie éprouvée } \\
\text {-Moins coûteuse lorsque } \\
\text { utilisée sur le Web } \\
\text {-Format documentaire } \\
\text { universel }\end{array}$ & $\begin{array}{l}\text {-Technologie flexible } \\
\text {-Format documentaire } \\
\text { flexible }\end{array}$ & $\begin{array}{l}\text {-Utilisation sur le Web } \\
\text {-Facile d'implantation } \\
\text { lorsque présence d'un } \\
\text { intranet }\end{array}$ & -Rapidité \\
\hline Inconvénients & $\begin{array}{l}\text {-Technologie coûteuse } \\
\text { lorsque utilisée sur un } \\
\text { RVA } \\
\text {-Format documentaire } \\
\text { peu flexible }\end{array}$ & $\begin{array}{l}\text {-Technologie encore } \\
\text { émergente et peu éprou- } \\
\text { vée } \\
\text {-Coûts élevés }\end{array}$ & & $\begin{array}{l}\text {-Coûts de développement } \\
\text {-Généralement pas utilisé } \\
\text { sur le Web }\end{array}$ \\
\hline Risques & -Faibles & $\begin{array}{l}\text {-Élevés car technologie } \\
\text { récente }\end{array}$ & -Faibles & -Faibles \\
\hline
\end{tabular}




\section{Interdépendance réciproque : SIIO Réseau}

La troisième forme d'interdépendance étudiée est l'interdépendance réciproque. Il ne s'agit plus ici d'une relation séquentielle telle que vue dans le chapitre précédant mais d'une relation de type réseau entre les différents partenaires d'affaires. La relation se veut généralement un peu plus aléatoire, moins souvent prédéfinie et, par conséquent, plus difficile à modéliser. Ainsi les flux d'information entre les partenaires sont généralement beaucoup plus dynamiques.

\subsection{Introduction au travail en collaboration}

Dans notre contexte d'étude inter-organisationnel, cette relation se retrouve généralement dans des situations d'équipes virtuelles, de conception assistée par ordinateur (CAO) entre manufacturiers et sous-traitants dans un contexte de collaboration. Les systèmes d'information inter-organisationnels qui supportent le travail fait en collaboration sont regroupés sous les termes Groupware ou Computer-Supported Cooperative Work (CSCW). Ces technologies, initialement conçues pour être utilisées dans un contexte interne, servent de plus en plus dans les relations interorganisationnelles. Aussi, il s'agit, sans aucun doute, de la forme d'interdépendance pour laquelle le moins de solutions d'automatisation existent mais pour laquelle il y a un potentiel de développement important.

Typiquement, le premier niveau technologique pour ce type de systèmes consiste en l'utilisation du téléphone, du fax ou du courrier électronique et il correspond au niveau auquel de nombreuses organisations se situent encore à ce jour. À un stade plus avancé, on y retrouve les technologies qui permettent de supporter du travail réalisé de façon synchronisée telles que la vidéo-conférence ou le partage d'écran (desktop sharing) mais également le travail réalisé de façon asynchrone comme par exemple l'édition commune de documents et les systèmes de gestion de workflows.

Nous allons nous intéresser dans ce chapitre à trois technologies principales. D'autres ont leur importance, mais l'édition commune de documents, les systèmes de gestion de workflows ainsi que le partage d'écran sont beaucoup plus susceptibles d'être utilisés dans le support de processus d'affaires c'est à dire supportant le processus de production de plusieurs organisations.
Page 29

Dans le cas d'interdépendance réciproque, la relation entre les partenaires est de type réseau et les flux d'information sont beaucoup plus dynamiques. 


\subsection{Les systèmes de gestion de workflows}

Les systèmes de gestion de workflows sont des systèmes qui permettent de coordonner les différentes activités d'un processus de façon asynchrone. Le Workflow management coalition (WfMC) définit un workflow comme étant l'automatisation, partielle ou entière, ou la facilitation par informatisation d'un processus d'affaires.

Les systèmes de gestion de workflows permettent de coordonner les différentes activités d'un processus

d'affaires de façon asynchrone.

Un système de gestion de workflows permet de créer, de définir, le plus souvent graphiquement, et enfin d'exécuter des workflows. Cette exécution passe par l'assignation, pour chaque activité, des tâches aux personnes compétentes et par l'exécution des applications TI permettant d'automatiser l'activité dans son intégralité ou en partie lorsqu'une intervention humaine est nécessaire.

La figure 12 illustre l'utilisation d'un tel système dans le cas d'un processus d'approbation de crédit dans une banque. Le processus requiert trois interventions humaines. La première consiste à saisir les informations relatives à la demande. La seconde consiste pour un responsable de crédit à approuver le prêt et enfin la dernière activité consiste pour le gérant de la banque à donner son approbation finale.

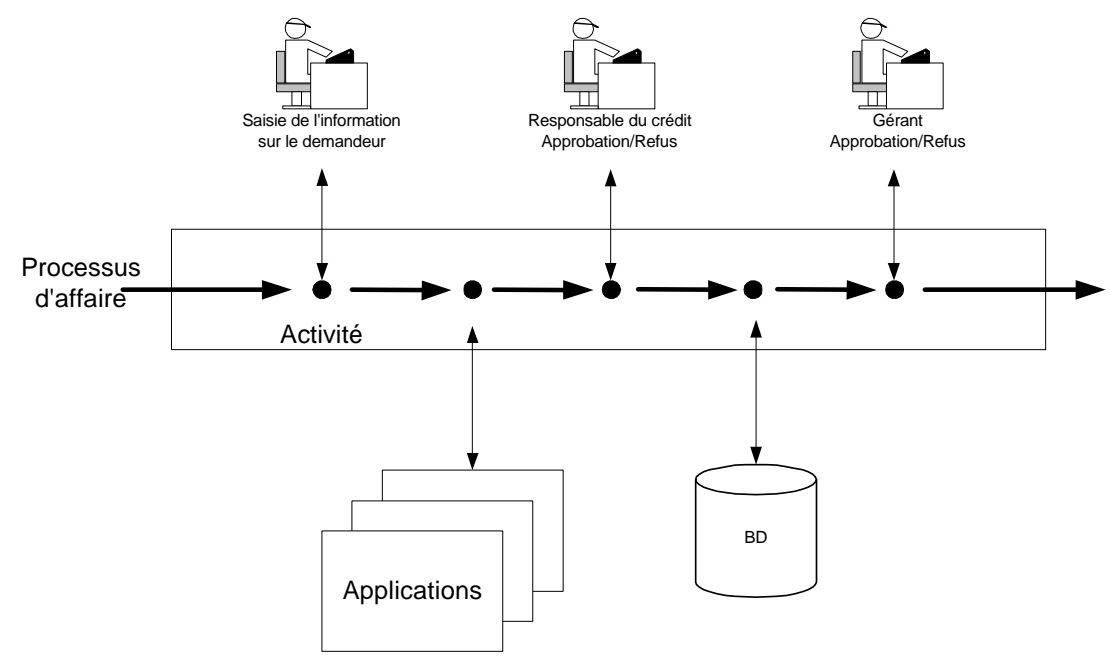

Figure 12 - Un système de gestion de workflows $^{8}$

${ }^{8}$ Adapté de Workflow Management Coalition, The Workflow Reference Model, Jan. 1995. 
Dans un tel cas, le système de gestion de workflows exécute de façon dynamique chaque activité en assignant à chaque individu les tâches qu'il doit compléter, et en exécutant les applications TI qui vont lui permettre de prendre une décision. Dans le cas du responsable du crédit, il pourrait s'agir d'une application d'aide à la décision qui illustrerait l'historique du client en terme de crédit et qui permettrait également de saisir la décision d'approuver ou non le crédit.

Les avantages d'un tel système à l'interne sont nombreux (assignation automatique des tâches, standardisation des processus, surveillance des instances...) mais leur utilisation récente dans un contexte inter-organisationnel a donné une nouvelle dimension à ces systèmes. En effet, les systèmes de gestion de workflows les plus récents sont maintenant combinés à des outils de traduction vers XML et à des outils d'intégration et servent maintenant de porte d'entrée vers les autres systèmes ou applications d'une organisation. Microsoft Biztalk Server est un exemple de produit de ce type.

La figure 13 illustre un tel système. En input, un tel système peut recevoir des documents sous une multitude de formats différents tels que XML ou EDI et les traduire sous des formats conformes pour les différents systèmes présents à l'intérieur de l'organisation tels que des fichiers plats (“flat files") pour les systèmes hérités, des IDocs pour SAP R/3 ou des messages pour des middlewares tels que Microsoft Message Queuing ou IBM MQSeries.

De plus, la configuration se fait généralement de façon graphique en modélisant le processus d'affaires supporté et en assignant des applications ou un envoi de message précis à chacune des activités. Ainsi, on modélise toujours le processus d'affaires graphiquement. Cependant, il ne s'agit plus vraiment de coordonner les activités d'individus mais plutôt celles des différents systèmes présents dans une organisation. La réception d'un document ou la transmission une commande à l'interne sert de déclenchement pour l'exécution d'un workflow dont les étapes seront automatisées, soit par l'appel d'applications (au format COM+ ou EJB par exemple), soit par l'envoi de documents ou de messages.
Le système de gestion de workflows est dynamique et peut recevoir des documents sous une multitude de formats différents tels que XML ou EDI et les traduire en formats compatibles avec les différents systèmes présents au sein de l'organisation. 


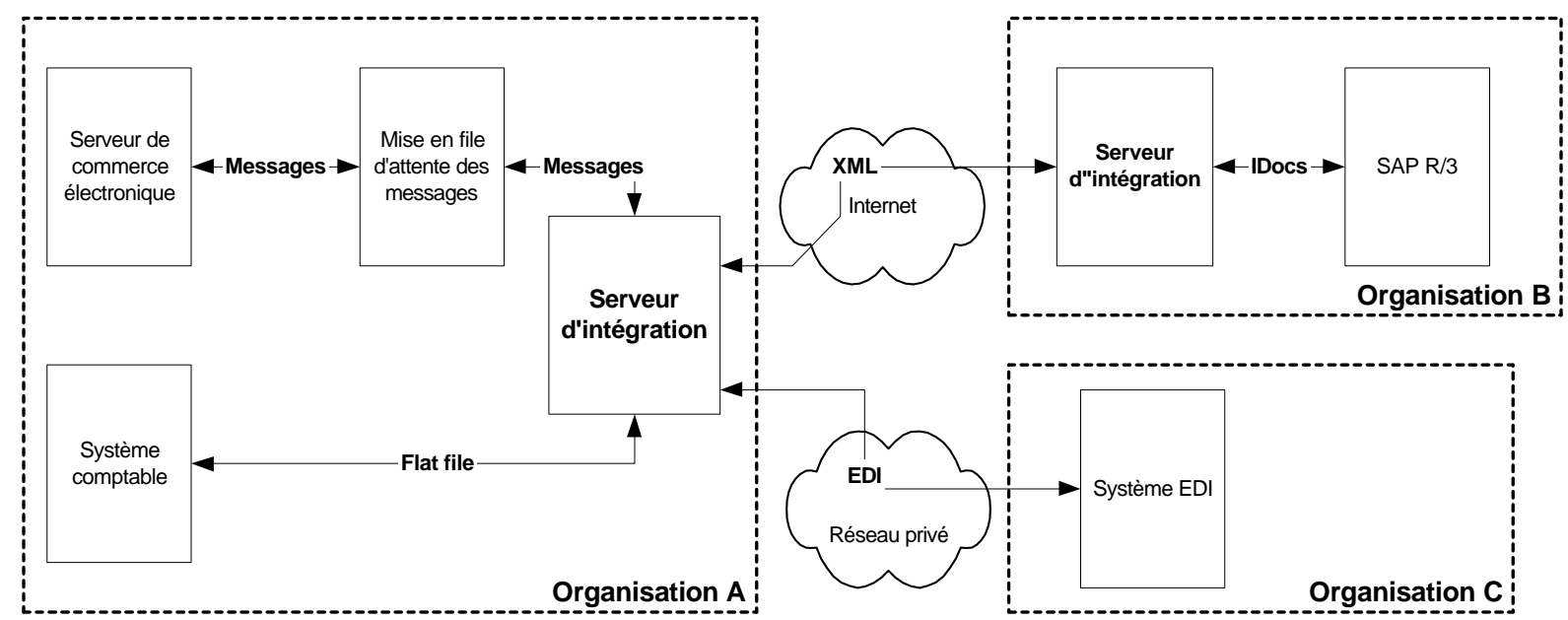

Figure 13 - Systèmes d'intégration

De tels systèmes sont particulièrement utiles lorsque la chaîne d'approvisionnement est complexe et se présente sous forme de réseau, mais également lorsqu'elle présente des environnements technologiques hétérogènes. L'entreprise Marks $\&$ Spencer qui souhaitait relier les informations sur ses ventes en magasin à son centre de données pour ensuite les transmettre à ses 500 fournisseurs a récemment développé un tel système XML. Il a ainsi permis l'intégration d'applications hétérogènes autant à l'interne (systèmes de ventes, comptables, d'inventaires) qu'à l'externe en permettant d'échanger sous divers formats avec ses fournisseurs.

Il faut noter que de tels systèmes nécessitent des investissements initiaux qui seront d'autant plus importants que les environnements à intégrer sont hétérogènes et que l'étendue des processus inter-organisationnels supportés sont importants.

\section{3 Édition commune de documents}

Nous venons de voir dans le précédent système que l'échange de documents permettait d'exécuter un workflow relié au processus de production de l'organisation. Néanmoins, il est important de noter qu'il existe également entre organisations un workflow de nature purement documentaire qui participe généralement peu au processus transactionnel de production en tant que tel. Il s'agit par exemple du flux de documents de contrôle de qualité ou de schémas techniques qui précèdent les premières prises de commandes entre un manufacturier et son sous-traitant. 
Pour gérer un tel flux, parfois complètement non défini à l'avance, on utilise souvent des serveurs de gestion documentaire ${ }^{9}$ qui permettent grâce à des outils de recherche et d'indexage d'accéder rapidement à l'information ou au document souhaité. Le stockage documentaire est généralement facilité par l'usage d'un format unique défini par exemple en XML. Originalement conçus pour être utilisés dans un contexte intra-organisationnel, ils trouvent maintenant leur place dans des contextes beaucoup plus vastes grâce à leur architecture web.

De plus, ils se combinent maintenant à des outils de travail en équipe permettant ainsi de créer un site web pour le travail en collaboration, de conduire des sondages et de réaliser des évaluations de documents sans toucher à leur forme initiale, mais également de s'intégrer à un ensemble d'applications de bureautique déjà présentes dans l'organisation.

\subsection{Synthèse des technologies}

Le tableau suivant présente une synthèse des deux technologies que nous venons de présenter. À nouveau, il est important de noter qu'il s'agit de technologies complémentaires puisqu'elles ne supportent les mêmes types de processus d'affaires.
Les serveurs de gestion documentaire, combinés à des outils de travail en équipe, permettent de créer des sites web qui facilitent la collaboration entre organisations.
Système de gestion de workflows et d'intégration

\begin{tabular}{|l|l|l|}
\hline Processus supportés & $\begin{array}{l}\text { - Processus d'affaires simples ou complexes } \\
\text { formalisés entre partenaires d'affaire }\end{array}$ & $\begin{array}{l}\text { - Processus d'affaires ad hoc (non } \\
\text { formalisés) }\end{array}$ \\
\hline $\begin{array}{l}\text { Environnements technologiques } \\
\text { (internes et externes) }\end{array}$ & - Multiples et complexes & - Accès par le Web \\
\hline Avantages & $\begin{array}{l}\text { - Permet l'intégration de technologies } \\
\text { différentes tout en se calquant au } \\
\text { processus d'affaires } \\
\text { - Supporte des relations d'affaires plus } \\
\text { complexes (de type réseau) }\end{array}$ & - Accès facile par le web \\
\hline Inconvénients & $\begin{array}{l}\text { - Faible coûts } \\
\text { - Investissement initiaux importants } \\
\text { - Technologie récente }\end{array}$ & \\
\hline
\end{tabular}

\footnotetext{
${ }^{9}$ tels que Documentum ou Microsoft SharePoint
} 


\section{Conclusion}

Avant d'implanter un SIIO, trois types de considérations à prendre en compte :

- Opérationnel

- Stratégique

- Risques associés

\section{Considérations}

opérationnelles

- Sécurité

- Intégration et systèmes patrimoniaux

- Intégration des processus et réingénierie
Ce rapport a présenté plusieurs types de systèmes d'information interorganisationnels qui permettent de supporter les processus d'affaires correspondants. En guise de conclusion, nous allons présenter un ensemble de considérations à prendre en compte lorsque l'on envisage d'implanter un SIIO ou de joindre les systèmes d'une organisation à un système inter-organisationnel existant. Ces considérations sont de trois ordres : opérationnel, stratégique, et risques associés.

\subsection{Considérations opérationnelles}

\section{- sécurité}

À quelles informations les partenaires auront-ils accès sur le système? Est-ce que la confidentialité de mes transactions est assurée? Ces questions doivent être soulevées et évaluées pour décider avant de joindre ou de mettre en place un SIIO.

Le problème de la sécurité se situe à différents niveaux dans le cas des SIIO. Pour un partenaire potentiel, le premier problème consiste à savoir quelle sera la nature des informations qu'il devra partager avec les autres organisations utilisant le système. Cette inquiétude est particulièrement présente dans le cas des places de marché électroniques. En effet, une des premières hésitations à en joindre une concerne la confidentialité des informations qui y seront acheminées. De plus, même si le système établit des règles d'accès et de partage de l'information disponible qui sont acceptables pour tous les partenaires d'affaires, une intrusion dans le système reste possible et pourrait révéler des informations commerciales utiles à un concurrent telles que les prix ou les quantités vendues.

\section{- Intégration et systèmes patrimoniaux}

Le véritable défi technologique pour un SIIO ne se situe pas véritablement au niveau de l'intégration entre entreprises mais plutôt au niveau de l'intégration avec l'ensemble des systèmes internes patrimoniaux. S'il peut être relativement simple de mettre en place un système d'échange de messages tel que EDI ou XML, la difficulté de l'intégration s'accroît avec le nombre de technologies différentes présentes à l'interne auxquelles il faudra connecter le système. En fait, d'un point de vue purement technologique, l'intégration d'un SIIO à l'interne présentera autant, si ce n'est plus de défis, que l'intégration à l'externe. 


\section{- Intégration des processus et réingénierie}

Tel que nous l'avons présenté plus tôt, la chaîne d'approvisionnement est essentiellement menée par le consommateur final. C'est sa décision d'achat en fin de chaîne qui va enclencher le long processus de production partant du premier fournisseur qui devra savoir quelles sont les préférence du consommateur en fin de chaîne. Cette dernière peut se situer jusqu'à une dizaine d'organisations en aval.

L'utilisation d'un système d'information inter-organisationnel permettra d'accélérer en grande partie ce processus par une automatisation des échanges mais encore bien souvent néglige-t-on de prendre en compte les opportunités de réingénierie apportées par les technologies de l'information dans une relation de chaîne d'approvisionnement ${ }^{10}$.

\subsection{Considérations stratégiques}

\section{- Objectifs communs}

Pour qu'un SIIO soit viable, chaque organisation doit avoir des objectifs communs vis-à-vis du système. Par exemple, dans le cas de la place de marché de l'automobile COVISINT, les constructeurs et leurs sous-traitants de premier niveau ont pour objectif collectif de réduire les coûts associés à la production d'un véhicule ainsi que le temps nécessaire à sa mise en marché. Cette convergence a permis de fixer des objectifs clairs et non-ambigus au système. Prenons maintenant le cas de CIS$\mathrm{CO}$, fabricant de composants en réseautique, dont l'objectif est d'éliminer les intermédiaires en faisant de la vente directe sur le web à ses clients corporatifs. Il devient rapidement évident que l'utilité d'un SIIO complexe qui le relierait à un distributeur (dont l'objectif serait de servir d'agrégateur sur le marché) est peu élevée.

\footnotetext{
${ }^{10}$ DELL est un exemple d'organisation qui a réussi à utiliser les systèmes d'information inter-organisationnels dans cette optique. La problématique était simple et consistait à trouver un moyen plus rapide pour faire parvenir les ordinateurs commandés à ses clients. L'entreprise décida alors de ne faire ses achats de moniteurs qu'auprès d'un seul fournisseur, SONY en l'occurrence. De plus, plutôt que de devoir commander le moniteur et de l'ajouter au reste de l'envoi, DELL chargea ses compagnies de livraison (Airbone Express et UPS) de faire l'assemblage entre le moniteur venant du Mexique et le reste des composants provenant d'Austin au Texas, la coordination se faisant grâce à des systèmes d'échange de données. Ainsi, on a supprimé les étapes de réception du moniteur et d'assemblage de la commande et supprimé un envoi dans la chaîne d'approvisionnement.
}

Considérations stratégiques

- Objectifs communs

- Alignement du système avec la stratégie d'entreprise

- Conflits entre les canaux de distribution 


\section{- Alignement du système avec la stratégie d'entreprise}

En plus de correspondre à des objectifs collectifs, le système devra également s'aligner sur la stratégie d'affaires de chaque organisation qui souhaite l'utiliser. À titre d'exemple, certains constructeurs automobiles ont refusé de joindre COVISINT, trouvant le système trop orienté vers une collaboration étroite entre les partenaires. Cette vision est particulièrement en contradiction avec la stratégie d'une firme telle que BMW qui préfère utiliser des pièces faites de façon unique pour ses véhicules plutôt que des équipements génériques, comme le font les constructeurs américains.

\section{- Conflits entre les canaux de distribution}

Tel que nous l'avons présenté dans la figure 2. Un nombre important de chemins distincts existe entre la matière première et le consommateur final. Ces différents chemins se définissent comme des canaux de distribution différents. Ces canaux peuvent être différenciés par un fournisseur utilisé différent ou par une technologie inter-organisationnelle différente. En effet, nous avons vu que dans une relation client-fournisseur, on pouvait trouver une complémentarité parmi les différentes technologies.

Néanmoins, lorsque les mêmes informations sont transmises par deux canaux différents, la situation d'un conflit de canaux de distribution peut survenir. On peut ainsi obtenir un prix d'achat différent à partir de deux systèmes inter-organisationnels différents ou obtenir un prix différent entre le système, extranet par exemple, qui permet une relation directe entre le client et le fournisseur, celui d'un distributeur. Cette situation peut être très dommageable pour la relation de l'entreprise avec son client.

Les exemples de firmes telles que CISCO et DELL qui ont réussi à générer des volumes de ventes importants via des canaux de distribution web ont incité beaucoup d'entreprises à tenter d'en faire autant et à espérer ainsi une désintermédiation entre eux et leurs consommateurs. Dans certains cas, on peut obtenir des résultats intéressants, notamment lorsque la stratégie est de type " Click and Mortar" combinant la prise de commande sur le Web tout en gardant un lieu de service physique. Dans d'autres cas, le résultat peut être plus difficile. Levi Strauss par exemple a fini par 
fermer son site web, notamment parce que ses ventes endommageaient la relation que l'entreprise entretenait avec certains de ses distributeurs qui s'étaient vu interdire le droit de vendre sur le web. En fait, bien souvent, les profits générés par une vente directe sans intermédiaire peuvent être effacés par la perte d'un intermédiaire efficient dans le canal de distribution.

Dans une relation de chaîne d'approvisionnement, ce résultat peut être particulièrement dangereux pour une organisation. Il convient donc, avant de se lancer dans la mise en place d'un système d'information inter-organisationnel, de déterminer avant tout si nos clients vont en profiter ou non. En fait, la définition d'un canal de distribution doit se faire avec notre client en perspective et c'est dans cette même optique que doit se faire la réingénierie d'un processus inter-organisationnel.

\subsection{Risques associés}

\section{- Confiance et conflits}

Nous avons présenté, dans ce rapport, une typologie des SIIO en fonction du type d'inter-connectivité. Il existe une possibilité de conflit qui pourrait être associée à chacune des formes de SIIO. La possibilité de conflit est une nouvelle considération à prendre en compte dans les SIIO. En effet, une incompatibilité de culture organisationnelle, des conflits de personnalité entre dirigeants ou une volonté de domination importante du marché au point d'être contraignante pour nos " partenaires" pourraient mettre en danger l'efficacité de n'importe quel SIIO, même si ce dernier s'avérait techniquement parfait. Quelques exemples de risque par nature du système sont cités plus bas.

Dans les SIIO partagés, des situations de conflit peuvent arriver lorsqu'un des membres du SI partagé abuse des ressources au point de réduire la qualité du SI pour les autres membres du système. Un des membre pourrait également tenter d'utiliser l'information collective dans un but personnel ou de surveiller les activités de ses concurrents, comme ce fut le cas pour les systèmes de réservation partagés qui avaient été créés par une compagnie aérienne (SABRE et American Airlines). Par conséquent, il pourrait être plus sûr de donner le contrôle d'un tel système à un intermédiaire neutre. Néanmoins, les SI partagés minimisent les interactions directes entre les membres du système de par leur structure centralisée réduisant ainsi la
La définition d'un canal de distribution doit se faire avec le client en perspective et c'est dans cette même optique que doit se faire la réingénierie d'un processus inter-organisationnel. 
potentialité de conflit.

Dans une relation de chaîne d'approvisionnement, les sources de conflit pourront provenir d'un comportement opportuniste d'une organisation qui cesserait d'utiliser le système pour faire des transactions avec d'autres partenaires. Elles pourraient également venir d'asymétries d'informations entre partenaires. Par exemple, un distributeur pourrait fournir des informations privilégiées à ses fournisseurs qui accepteraient de collaborer selon leurs règles.

Enfin, dans un SIIO collaboratif, le nombre d'interactions directes et la complexité des rapports augmentent la probabilité de conflit. Par exemple, le rejet d'un document de contrôle de qualité sur un système de gestion de workflow, des demandes de révisions d'un schéma technique par un manufacturier à son sous-traitant sur un système de gestion documentaire sont tant de situations qui, de par la nature même

\section{Risques associés}

- Le nombre d'interactions directes et la complexité des rapports augmentent la probabilité de conflit.

- Les investissements réalisés pour la mise en place d'un SIIO peuvent mettre les partenaires dans une position de lock-in. de la collaboration, sont susceptibles de mener à un plus grand nombre de divergences de points de vue qu'un système transactionnel EDI qui ne fait qu'acheminer des factures.

Un certain niveau de confiance est nécessaire pour la mise en place d'un SIIO. Ce niveau va différer en fonction du type de système choisi. Il est important de noter que le SIIO peut également, en formalisant la collaboration, réduire la potentialité de conflits.

\section{- Lock-in}

Le lock-in est une arme à deux tranchants. Il s'agit d'une situation qui rend difficile un retour en arrière dans la relation que l'on a avec nos partenaires en raison de l'importance des investissements réalisés pour mettre en place le SIIO. Par exemple, dans le cas d'un système EDI utilisé dans une relation de chaîne d'approvisionnement, on est plus ou moins contraint de faire affaire avec le fournisseur auquel on est relié. On est donc également en partie "lié " à sa politique de prix. Mais, à l'inverse, de nombreux gains peuvent être réalisés par une telle situation puisque la collaboration entre les deux firmes devient beaucoup plus étroite. 


\subsection{Remarques finales}

Le véritable défi pour l'implantation d'un SIIO ne se situe généralement pas à un niveau technologique. Des différences au niveau des objectifs, un mauvais alignement avec les différentes stratégies d'affaires, un manque de confiance ou le refus d'une situation dite de "lock-in ", c'est-à-dire d'ancrage à un partenaire d'affaires pourront mener à un échec rapide du système, quelle que soit sa qualité technologique.

L'utilisation d'un SIIO peut permettre d'ouvrir un nouveau canal de distribution. Néanmoins, il faut faire attention à ne pas nuire aux relations commerciales avec des partenaires existants. La vente directe, par exemple, pourrait vous aliéner vos meilleurs distributeurs et coûter cher à court terme au niveau des ventes.

L'utilisation qui est faite du système pourrait dégager un avantage compétitif. Ainsi, une vitesse d'acheminement des commandes supérieure grâce à un traitement rapide des messages reçus en format EDI, des possibilités de personnalisation avancées offerte sur un Extranet grâce à un processus de production original ou des fonctionnalités supérieures grâces à des services Web originaux sont tant de moyens qui permettraient d'obtenir un avantage compétitif au travers de l'utilisation d'un SIIO.

Un SIIO ne fournira pas nécessairement un avantage compétitif à votre organisation. Il peut s'agir d'un système dont l'utilisation est largement généralisée dans de nombreuses industries. Dans ce cas, le système devient bien plus une nécessité stratégique qu'un avantage. C'est un système dont on ne peut se passer mais qui ne différencie pas la compagnie qui l'utilise.

Lorsqu'on envisage la mise en place d'un SIIO dans une relation de chaîne d'approvisionnement, il est important, au-delà des gains d'efficacité qui seront faits avec notre partenaire d'affaires direct, de déterminer comment ces gains se répercuteront sur le consommateur final. Trop souvent, on oublie que c'est sa décision d'achat qui soutient l'ensemble de la chaîne. Les décisions doivent donc tenir compte de l'ensemble de la chaîne, et idéalement rester avantageuses pour tous les partenaires d'affaires.
Lorsqu' on envisage la mise en place d'un SIIO, il est important, au-delà des gains d'efficacité qui seront faits avec notre partenaire d'affaires direct, de déterminer comment ces gains se répercuteront sur le consommateur final. 


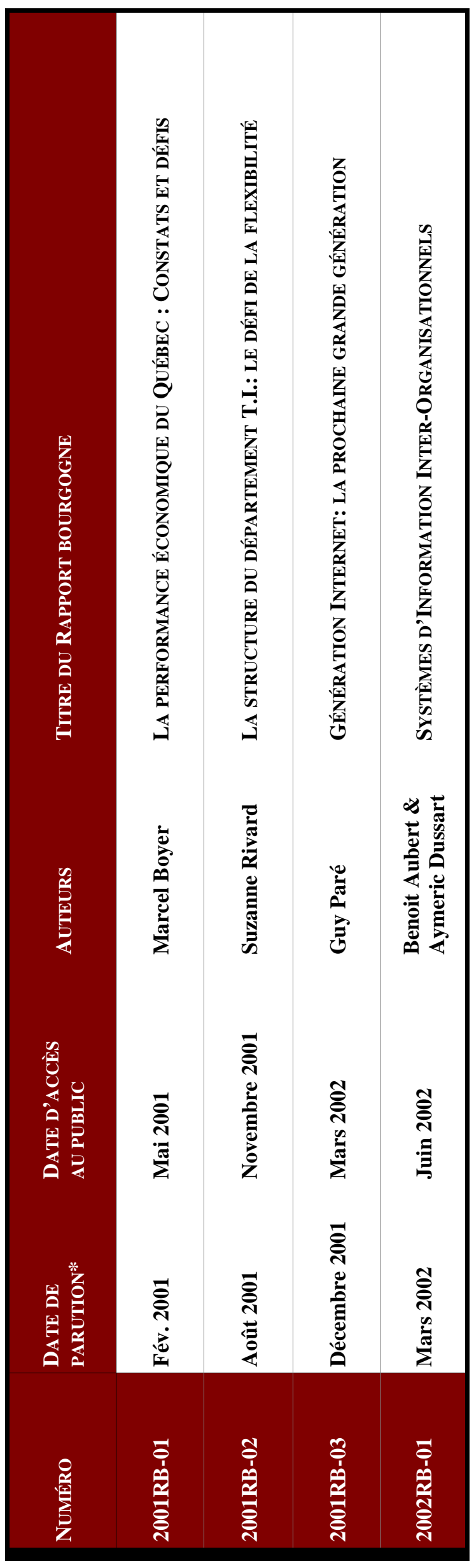

